\title{
Realization of Long Reach High speed Optical System by Incorporating Optical Phase Conjugation (OPC) Scheme
}

\author{
Vishal Sharma, $\mathrm{PhD}$ \\ Affiliated to IK PTU, Jalandhar, Punjab, India \\ Shaheed Bhagat Singh State Technical Campus, \\ Ferozepur, Punjab, India
}

\author{
Ruchi Sharma \\ Affiliated to IK PTU, Jalandhar, Punjab, India \\ Shaheed Bhagat Singh State Technical Campus, \\ Ferozepur, Punjab, India
}

\begin{abstract}
This work reports the lessening of optical signal distortion due to group velocity dispersion by incorporating the optical phase conjugation (OPC) scheme in order to meet the requirement of high speed signals with better performance. The work reports a comparative discussion of with- and withoutincorporating OPC at varied values of its efficiency based on various performance measures such as BER, eye opening, Qfactor. Furthermore, along with the impact of OPC efficiency, the influence of laser line-width is also deliberated to realize a high speed optical system.
\end{abstract}

\section{Keywords}

Optical Phase Conjugation technique (OPC), BER, eye diagram, Q-factor.

\section{INTRODUCTION}

The communication system must dispense higher rate to speak the longest doable distance and most doable information rates with lowest bit error rates. The fiber based communication networks fulfill these needs together with minute attenuation loss and higher QoS [1],[2]. The upper information transmission rate operation causes inter-symbol interference owing to dispersion and fiber non-linearity. Variety of makes an attempt is initiated to beat this drawback to understand high speed optical links for previous years. Out of variety of techniques, Fiber Bragg grating (FBG) and Optical part conjugation (OPC) square measure principally adopted to compensate the link-loss owing to dispersion and fiber nonlinearity [3],[4],[5],[6]. Over the last three decades, optical phase conjugation (OPC) has been one of the major research subjects in the field of nonlinear optics and quantum electronics [7].OPC defines usually a special relationship between two coherent optical beams propagating in opposite directions with reversed wave front and identical transverse amplitude distributions. The unique feature of a pair of phaseconjugate beams is that the aberration influence imposed on the forward beam passed through an inhomogeneous or disturbing medium can be automatically removed for the backward beam passed through the same disturbing medium [8]. OPC also can be used for the cancelation of nonlinearities ensuing from the Kerr result like self-phase- modulation (SPM), cross-phase-modulation (XPM),intra-channel nonlinearities and nonlinear part noise [9],[10]11]. OPC conjointly plays imperative role so as to enhance the performance of coherent optical orthogonal frequency division multiplexing (CO-OFDM) communication systems and is investigated by formulating the four-wave mixture (FWM) method by suggests that of two/ 3 OPCs [12]. In quasi-linear systems, periodic dispersion compensation techniques square measure introduced to manage dispersive property of fiber together with fiber [13],[14]. For coherent detection, the nonlinear impairments may be eliminated by electrical effort mistreatment DSP technique at the receiver finish. Further, a technique is planned to partly make amends for nonlinear part noise (NPN) by subtracting the received part proportional to the received intensity to make amends for inter- and intra-channel non-linearities in several transmission systems [15].

\section{SIMULATIVE SETUP \& RESULT ANALYSIS}

A modulated $10 \mathrm{Gbps}$ NRZ information signal with a considerably high optical power is transmitted over a primary lossless fiber-link. The entire system consists of four basic elements: a transmitter, a fiber link, an OPC and a receiver. The NRZ signal produced at the transmitter side is passed over an optical splitter which is an integrated waveguide optical power distribution device and couples the transmission signal to the optical fiber link. This coupled signal passes through the cable which is assumed to be lossless in this model. After the signal travels for $1000 \mathrm{~km}$, an optical power meter is installed in the link to measure the power of the incoming signal. Further, the signal is passed through an OPC and after travelling through another splitter and optical link, it finally reaches the receiver where BER and $Q$ value of the received signal is measured along with the other measurements. In this section, eye diagrams before and after using an OPC are compared for an OPC incorporated linear optical system. Analysis of Q value is done along with the evaluation of Eye opening and BER at mean decision threshold for OPC incorporated linear optical system at different values of OPC efficiency.

First, we examine the Eye diagram for the system without using an OPC after the NRZ 10 Gbps signal travels for 1000 $\mathrm{km}$. As it is obvious from Figure 1.1, the eye of the signal received is completely closed and distorted thus suggesting signal loss due to non linear factors and chromatic dispersion. The amount of Distortion, jitter and Time variation of zero crossing is quite undesirably high while there is no definitive sampling point to determine the SNR. The signal received cannot be used for doing any further analysis hence implying a need to incorporate an OPC in the system. 


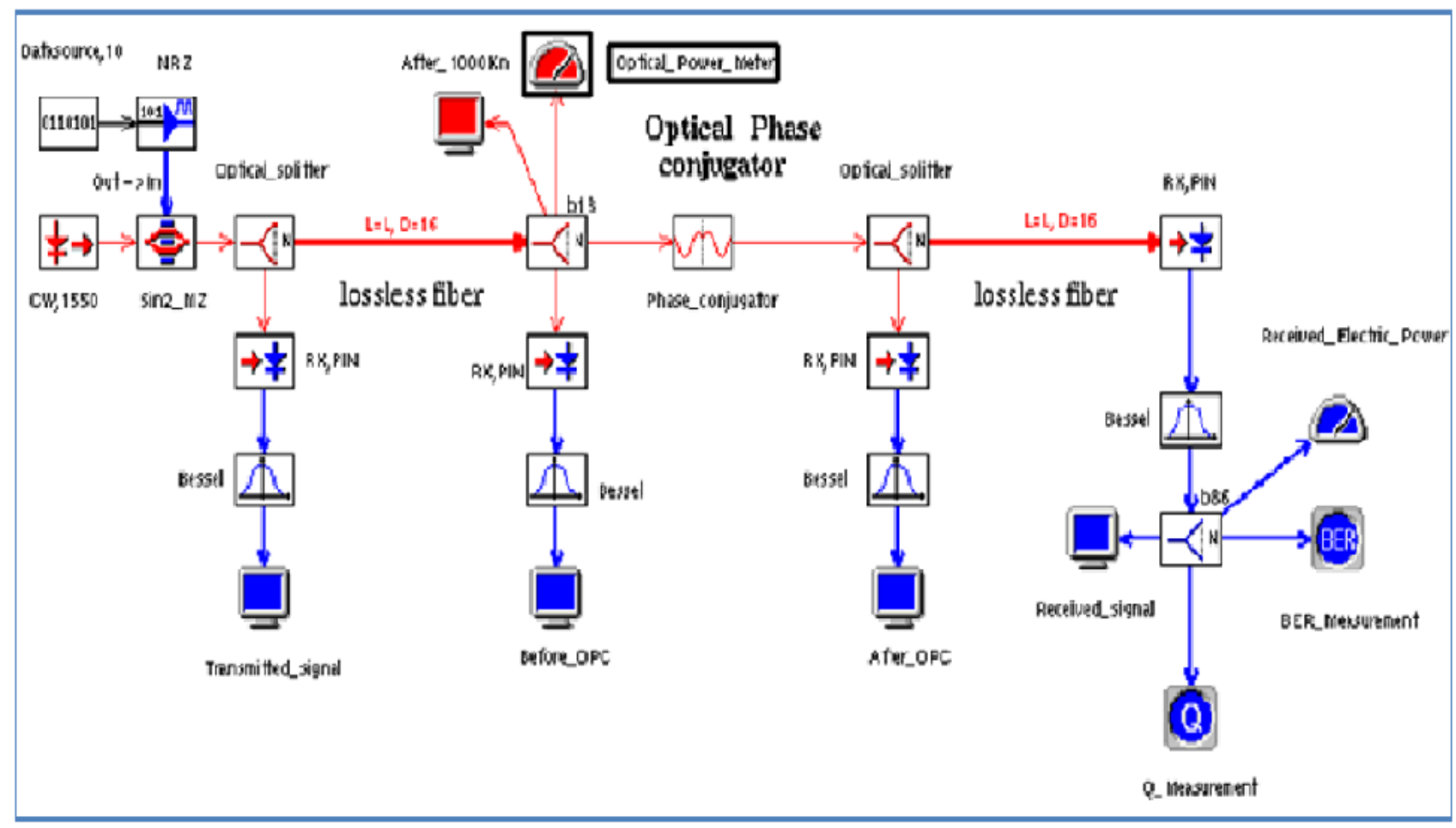

Figure 1: Simulative setup of an OPC incorporated linear optical system

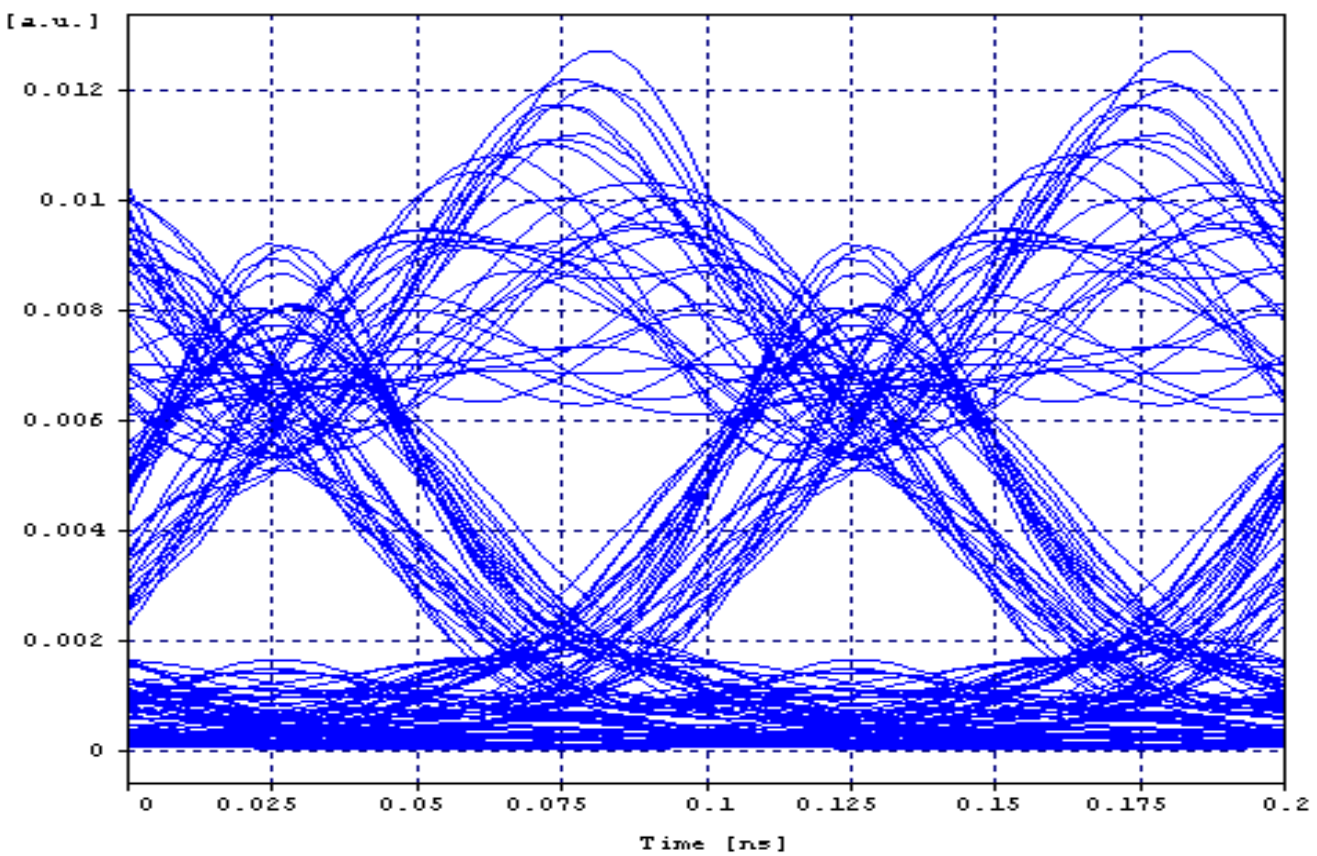

Figure 1.1: Eye diagram for an OPC incorporated linear optical system before using an OPC

Next, eye diagrams for an OPC incorporated linear optical system are analyzed for an optical span of $1000 \mathrm{Km}$ with varied OPC Efficiencies. As can be seen from Figure 1.2 (a), where the OPC efficiency is considered to be $50 \%$, there is an appreciable improvement in the eye diagram from as compared to the previous eye diagram of the received signal with no OPC. The opening of eye is dramatically increased hence making analysis of SNR and other parameters easier. Though the rise and fall times of the diagram are not quite finite, yet it provides a clear margin for such analysis. Figure 1.2 (b) reveals a further improvement in the eye opening when the OPC efficiency is taken to be $60 \%$. Similarly this improvement is noted for an OPC efficiency of $70 \%$ and $100 \%$ which can be clearly seen from Figure 1.2 (c) and (d). The eye is maximum relaxed at $100 \%$ efficiency providing an appreciable SNR and minimum values of interference and crosstalk. 


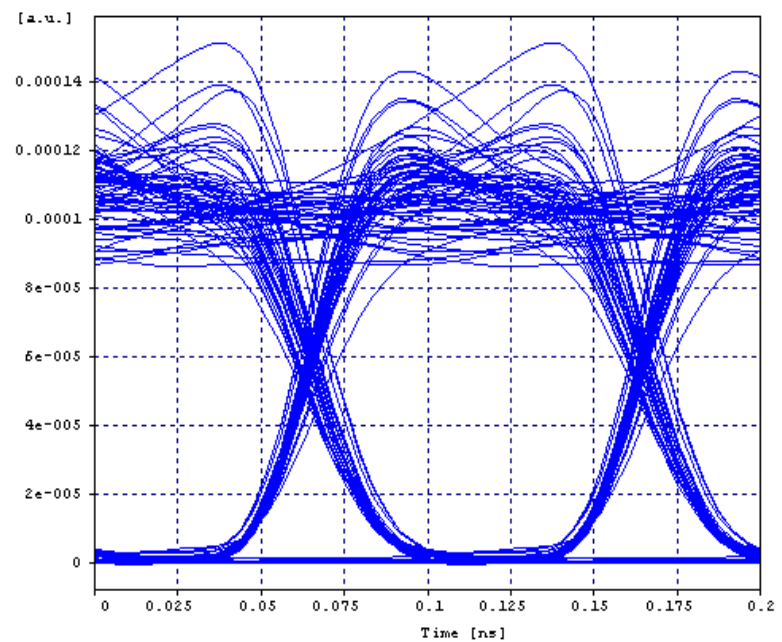

(a)

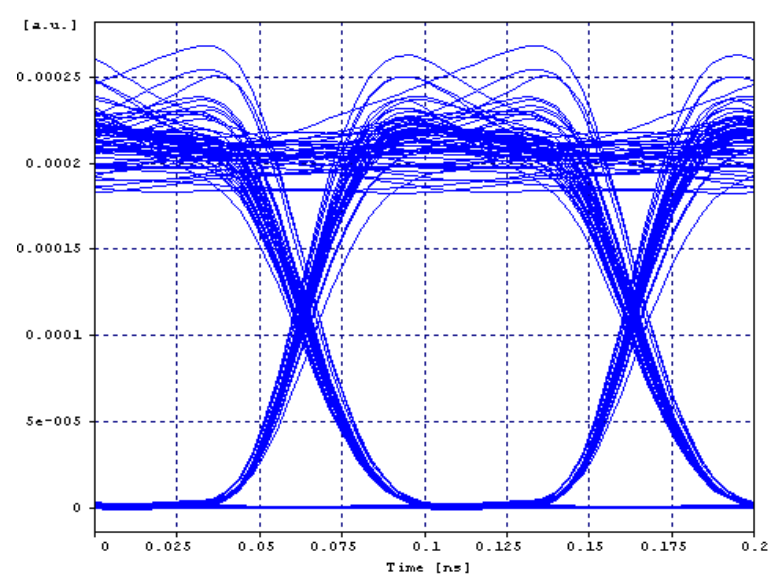

(c)

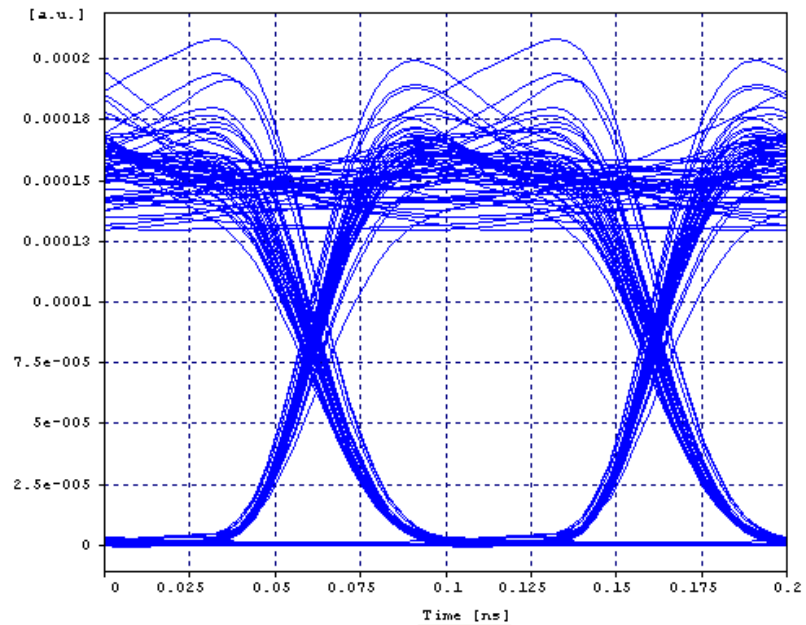

(b)

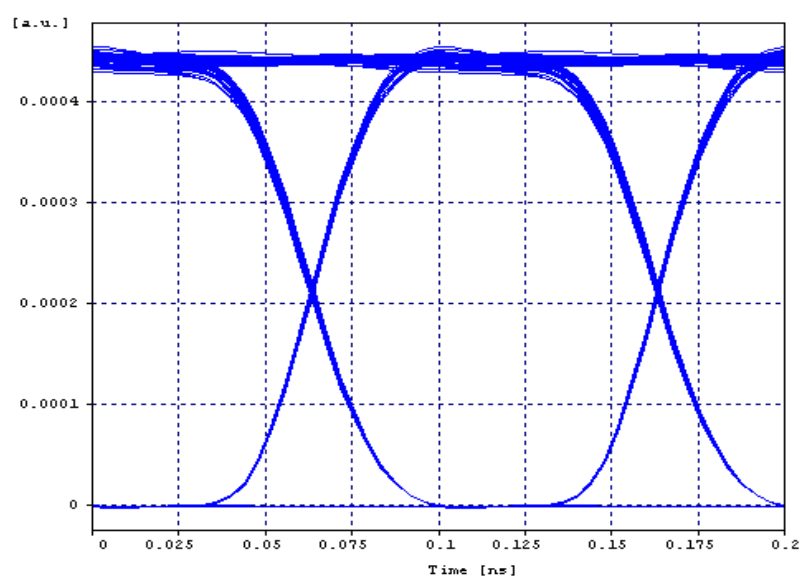

(d)

Figure 1.2: Eye diagram for an OPC incorporated Linear optical system after an optical span of $1000 \mathrm{Km}$ with OPC Efficiency of (a) $50 \%$, (b) $60 \%$, (c) $70 \%$, and (d) $100 \%$ at phase shift of 1.57

Further, Q value, Eye opening and BER at mean decision threshold are determined by taking three different values of OPC efficiency i.e. 0.5, 0.6 and 0.7. It is noted that the values of $\mathrm{Q}$ value and Eye opening decrease with the increasing Optical span while BER increases with the increase in the Optical span. The Q value at $500 \mathrm{~km}$ at OPC efficiency of 0.5 , 0.6 and 0.7 is noted to be 27,28 and 30 . These values go on decreasing and come to the values of 20,22 and 23 at the maximum Optical length of $1000 \mathrm{~km}$. The values of BER go from as low as 1e-040 to as high as 1e-010 for different values of OPC efficiency at different optical spans as can be seen from Figure 4.Figure 5 depicts the variation of $Q$ value with length when laser line-width is taken to be $100 \mathrm{KHZ}$ and 10 MHZ. The system shows a higher $Q$ value for the former consideration. 


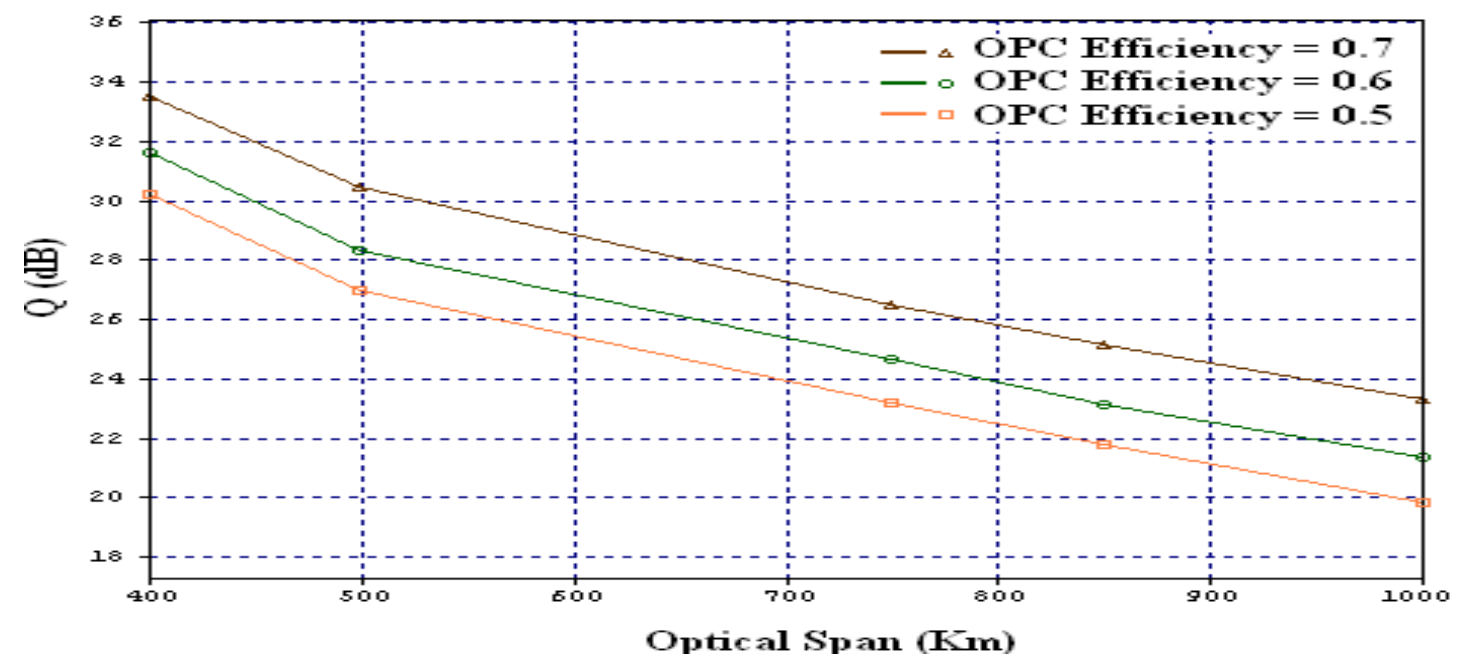

Figure 2: Evaluation of $Q$ value for an OPC incorporated linear optical system at varied values of OPC Efficiency

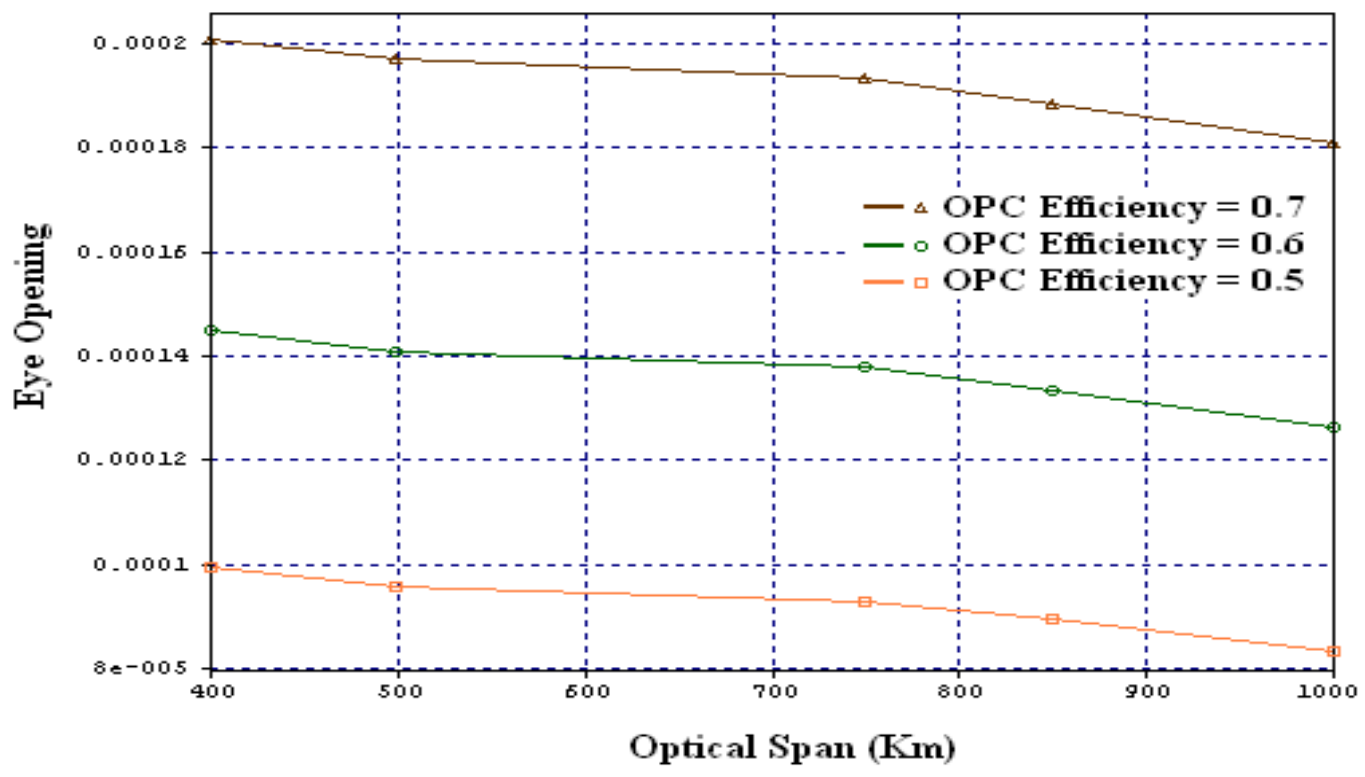

Figure 3: Evaluation of Eye opening for an OPC incorporated linear optical system at varied values of OPC Efficiency

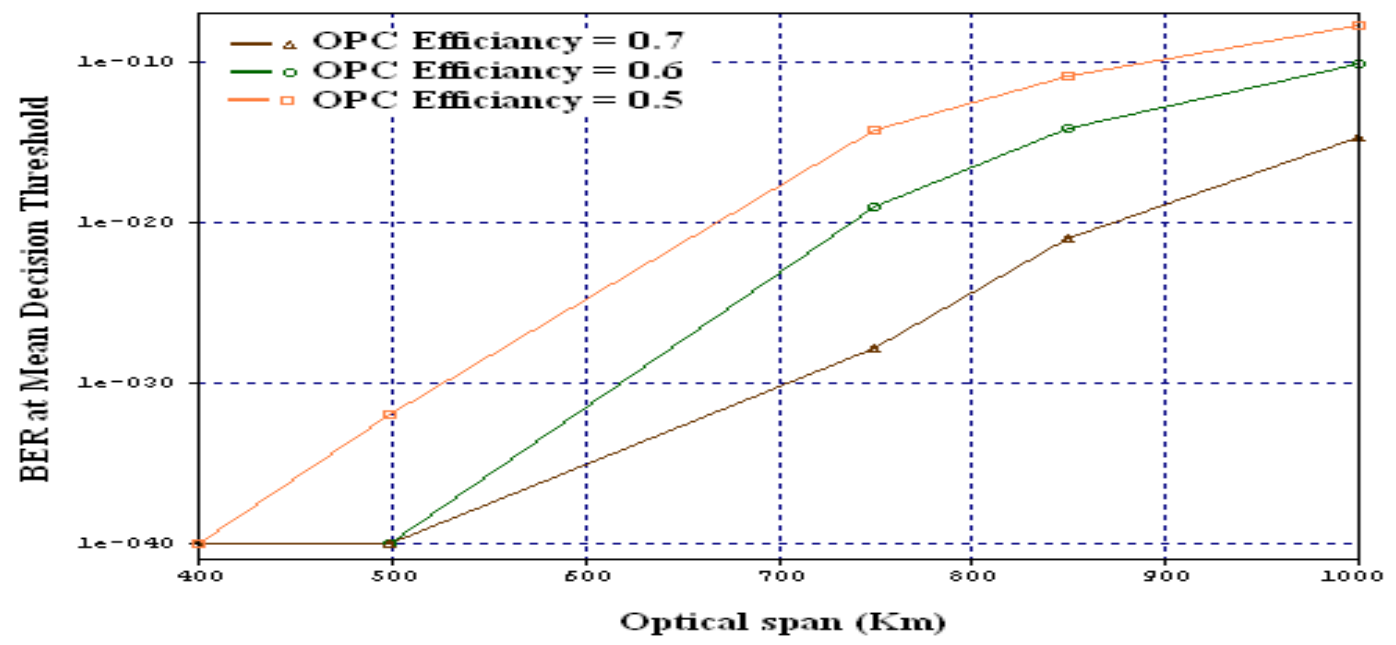

Figure 4: Evaluation of BER at mean decision threshold for an OPC incorporated linear optical system at varied values of OPC Efficiency 


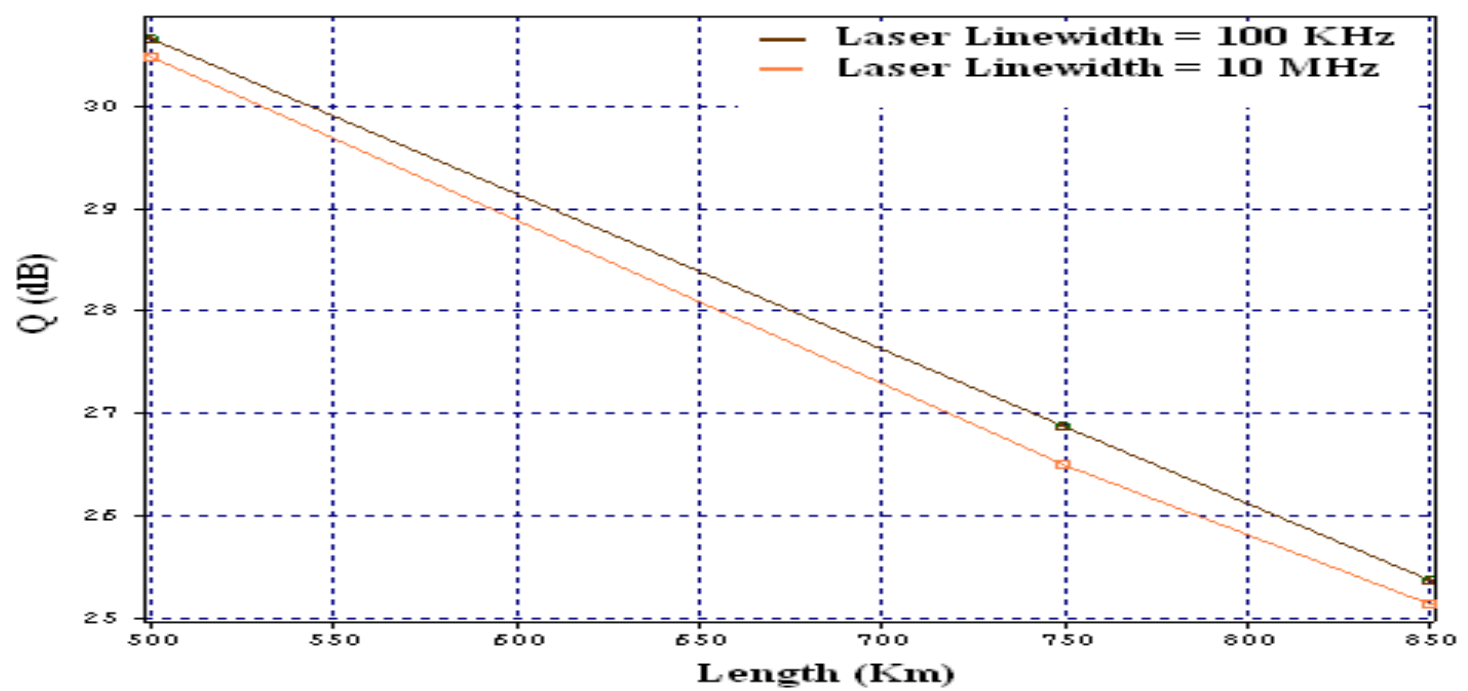

Figure 5: Evaluation of Q value at OPC Efficiency of $70 \%$ at varied values of Laser line-width

Evaluation of $\mathrm{Q}$ value BER at mean decision threshold at OPC Efficiency of $70 \%$ at varied values of laser power levels is done and is shown Figures 6 and 7 at laser powers of $0 \mathrm{dBm}$ and $5 \mathrm{dBm}$. The $\mathrm{Q}$ value is seen to decrease while the BER at mean decision threshold is seen to increase with an increase in Optical span. Figure 8 reveals estimation of eye opening at laser powers of $0 \mathrm{dBm}$ and $3 \mathrm{dBm}$. It is noted that the value of eye opening remains almost constant throughout all across the considered optical length i.e. 0.0002 while there is a constant decrease in the value from 0.0005 to 0.0001 when the laser power is taken to be $3 \mathrm{dBm}$.

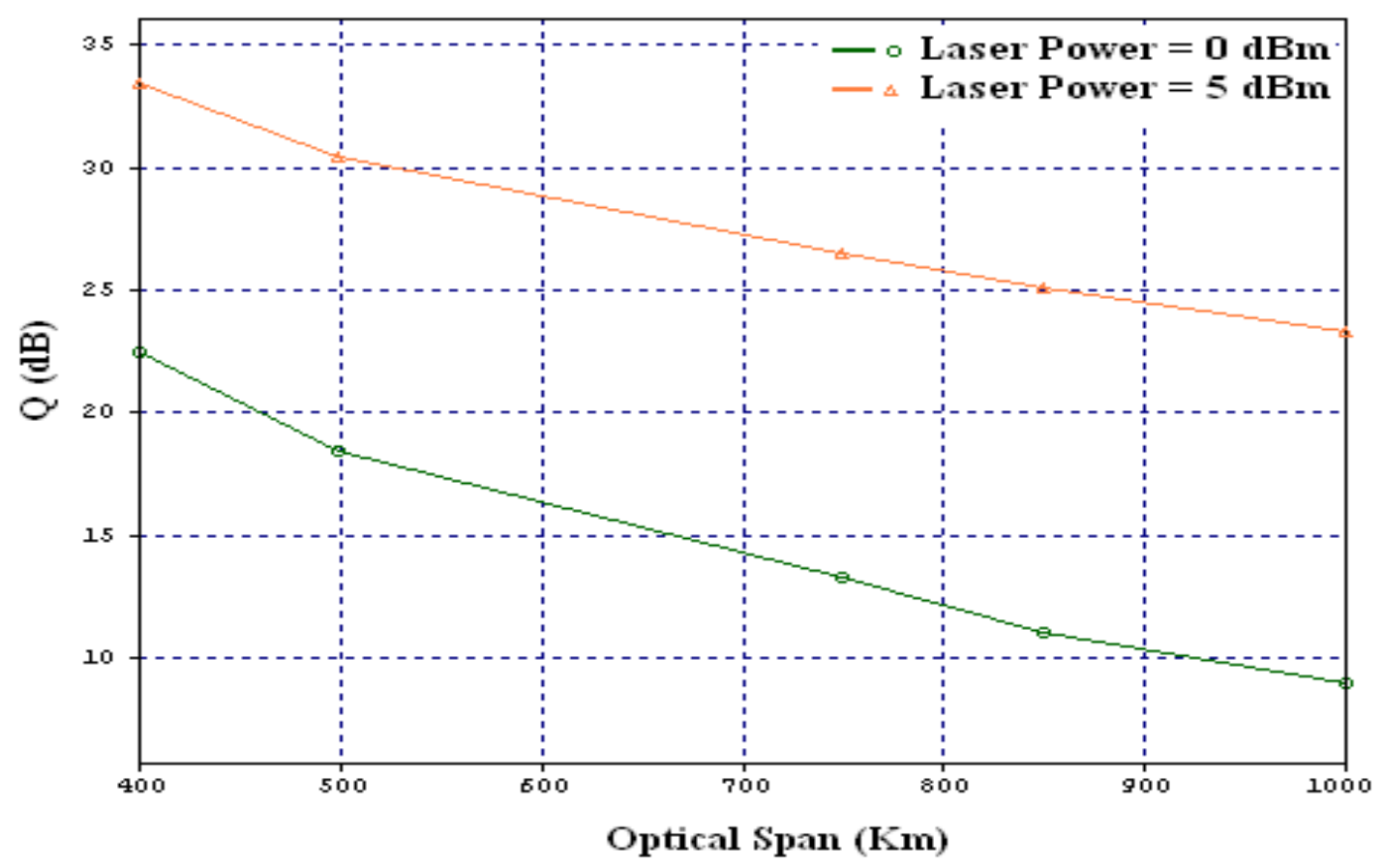

Figure 6: Evaluation of Q value at OPC Efficiency of 70\% at varied values of laser power levels 


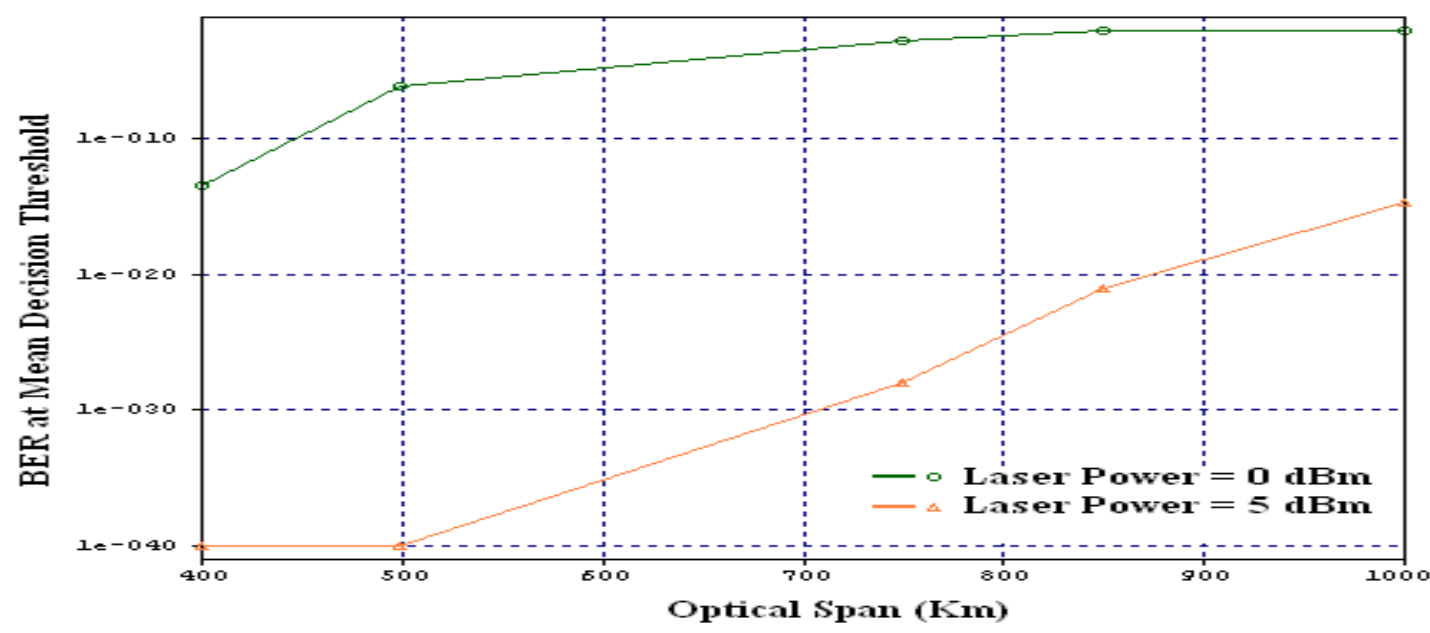

Figure 7: Evaluation of BER at mean decision threshold at OPC Efficiency of 70\% at varied values of laser power levels

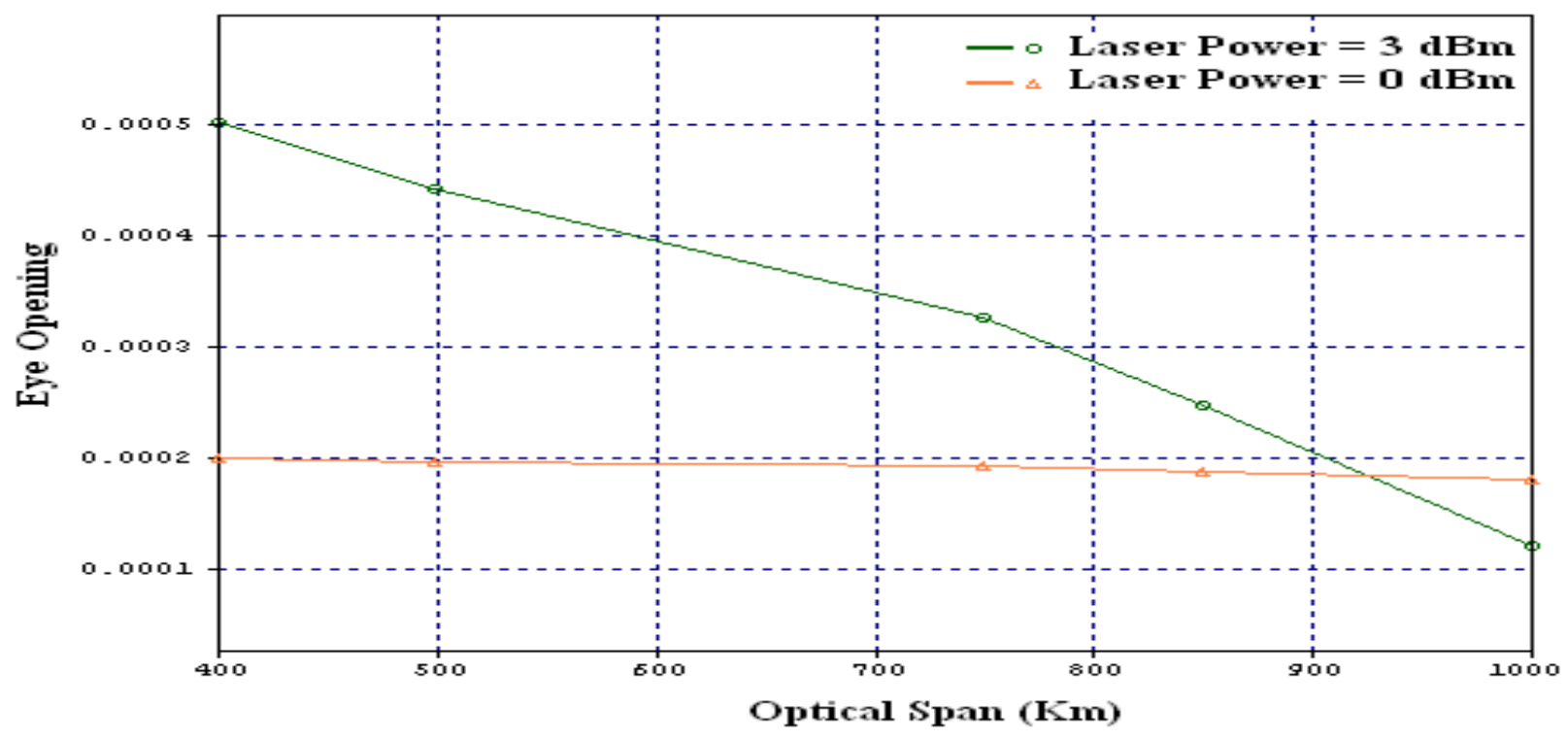

Figure 8: Evaluation of Eye opening at OPC Efficiency of $70 \%$ at varied values of laser power levels

\section{CONCLUSION}

In this paper, performance evaluation of an optical system is carried out for examining the influence of OPC technique to eliminate the dispersive effects. The system with- and without-OPC has been configured and are investigated using performance measures such as Q-factor, BER and eye opening. The observations reported in this work recommend the use of high efficient OPC in conjunction with less laser line-width to realize a long reach high speed optical system with good in-line dispersion compensation.

\section{REFERENCES}

[1] Gruner Nielsen L., "Dispersion compensating fibers", Journal of Light-wave Technology, pages: 3566-3579, volume 23, issue 11, Nov 2005.

[2] R.k Sethi and Aditya Goel, "Critical analysis of dispersion compensated optical communication system, International Journal of computer Application, (09758887) pages:1-14,Volume116 No. 15, April 2015.
[3] Kishore Bhowmik, Md. Maruf Ahammed and Md.Abdul Momin, "Reduction of dispersion in optical fiber communication by fiber bragg grating and optical phase conjugation technique", International Journal of Mobile Network Communication and Telematics, pages: 49-58, volume 2, issue 3, June 2012.

[4] Paolo Minzioni, Franscesco Alberti and Alessandro Schiffini, "Techniques for Non- linearity cancellation in embedded links by optical phase conjugation" ,Journal of light-wave Technology, pages: 2364-2370, volume 23, issue 8, August 2005.

[5] Jianqiang Li, Kun Xu, Guangtao Zhou, Jian Wu, Member, Jintong Lin, “ Dispersion-compensation schemes for $160-\mathrm{Gb} / \mathrm{s} 1200-\mathrm{km}$ transmission by optical phase conjugation", Journal of light-wave Technology, pages: 1986-1995, volume 25, issue 8, August, 2007.

[6] Smita S. Dabhade and Savita Bhosale, "Fiber bragg grating and phase conjugator as dispersion compensator", International Journal on Advanced 
Electrical and Electronics Engineering, (IJAEEE), pages: 15-19, volume 1, 2012.

[7] S. L. Jansen, D. van den Borne, M. Krummrich, S. Spalter and G.-D. Khoe, "Long-haul DWDM transmission systems employing optical phase conjugation", IEEE Journal of Selected Topics in Quantum Electronics, pages: 505520,Vol.12.No.,4July/August 2006.

[8] Hwang-Bin Yim1 and Seong-Real Lee2; "Dispersion managed optical links with optical phase conjugator placed at the various positions", International Journal of Multimedia and Ubiquitous Engineering, pages: 189198, volume 9, issue 12014.

[9] S. L. Jansen, D. van den Borne, C. C. Monsalve, S. Spälter, P. M. Krummrich, G. D. Khoe, and H. de Waardt, "Reduction of Gordon-Mollenauer phase noise by midlink spectral inversion," IEEE Photonics Technology, pages: 923-925, Vol.17.No.,4 April, 2005.

[10] S. L. Jansen, D. van den Borne, G. D. Khoe, H. de Waardt,C. C. Monsalve, S. Spalter, and P. M. Krummrich, "Reduction of phase noise by mid-link spectral inversion in a DPSK based transmission system," presented at the Optical Fiber communication Conference, pages1-3, Vol.4.No.,5,March 2005
[11] Morteza H. Shoreh, "Compensation of nonlinearity impairments in coherent optical OFDM system using multiple optical phase conjugate modules",Journal Optical Communication Network, Pages:549-558, Vol. 6 No. 6, June 2014.

[12] M.Faisal, Akihiro Maruta; "Mitigation of phase noise in dispersion compensated quasi-linear optical transmission systems", Optics Communications, Pages: 2430-2437, Vol No.26 January, 2010.

[13] Yan Gao, Fan Zhang, Liang Dou, Zhangyuan Chen, Anshi $\mathrm{Xu}$; "Intra-channel nonlinearities mitigation in pseudo-linear coherent QPSK transmission systems via nonlinear electrical equalizer", Optics Communications, Pages: 2421-2425, Vol No. 1March, 2009.

[14] M. Y. Hamza, S. Tariq and M. M. Awais, "Mitigation of the effects of self-phase modulation and group velocity dispersion in fiber optic communications: dispersion- and power-map co-optimization using the genetic algorithm", Journal Telecommunication Systems, pages: 451-459, Vol.55.No.,4April2014.

[15] Mohammad Faisal, Akihiro Maruta, "Mitigation of phase noise in dispersion compensated Quasi linear optical transmission systems", Optics Communications, Pages: 2430-2437, Vol No.283. 